\title{
Endobronchial ultrasonography with guide-sheath for peripheral pulmonary lesions
}

\author{
E. Kikuchi*, K. Yamazaki*, N. Sukoh\#, J. Kikuchi*, H. Asahina*, M. Imura*, Y. Onodera", N. Kurimoto ${ }^{+}$, \\ I. Kinoshita*, M. Nishimura*
}

Endobronchial ultrasonography with guide-sheath for peripheral pulmonary lesions. E. Kikuchi, K. Yamazaki, N. Sukoh, J. Kikuchi, H. Asahina, M. Imura, Y. Onodera, N. Kurimoto, I. Kinoshita, M. Nishimura. (C) ERS Journals Ltd 2004.

ABSTRACT: The usefulness of endobronchial ultrasonography (EBUS) with guidesheath (GS) as a guide for transbronchial biopsy (TBB) for diagnosing peripheral pulmonary lesions (PPL)s and for improving diagnostic accuracy was evaluated in this study.

EBUS-GS-guided TBB was performed in 24 patients with 24 PPLs of $\leqslant 30 \mathrm{~mm}$ in diameter (average diameter $=18.4 \mathrm{~mm}$ ). A $20-\mathrm{MHz}$ radial-type ultrasound probe, covered with GS was inserted via a working bronchoscope channel and advanced to the PPL in order to produce an EBUS image. The probe with the GS was confirmed to reach the lesion by EBUS imaging and $\mathrm{X}$-ray fluoroscopy. When the lesion was not identified on the EBUS image, the probe was removed and a curette was used to lead the GS to the lesion. After localising the lesion, the probe was removed, and TBB and bronchial brushing were performed via the GS.

Nineteen peripheral lesions $(79.2 \%)$ were visualised by EBUS. All patients whose PPLs were visible on EBUS images subsequently underwent an EBUS-GS-guided diagnostic procedure. A total of 14 lesions (58.3\%) were diagnosed. Even when restricted to PPLs $<20 \mathrm{~mm}$ in diameter, the diagnostic sensitivity was $53 \%$.

In conclusion, endobronchial ultrasonography with guide sheath-guided transbronchial biopsy was feasible and effective for diagnosing peripheral pulmonary lesions. Eur Respir J 2004; 24: 533-537.
*First Dept of Medicine, Hokkaido University School of Medicine, Sapporo, ${ }^{\#}$ Dept of Medicine, Iwamizawa Municipal General Hospital, Iwamizawa, and "Dept of Radiology, Hokkaido University School of Medicine, and ${ }^{+}$Dept of Surgery, National Hiroshima Hospital, Higashi-Hiroshima, Japan.

Correspondence: K. Yamazaki, First Dept of Medicine, Hokkaido University School of Medicine, North 15, West 7, Kitaku, Sapporo 060-8638, Japan.

Fax: 81117067899

E-mail:kyamazak@med.hokudai.ac.jp

Keywords: Bronchial brushing cytology endobronchial ultrasonography guide-sheath

peripheral pulmonary lesion primary lung cancer transbronchial biopsy

Received: December 152003

Accepted after revision: June 232004
The investigation of a peripheral pulmonary lesion (PPL) is a challenging diagnostic problem. A transbronchial approach using a fiberoptic bronchoscope (FB) under X-ray fluoroscopic guidance has been the most generally accepted method for diagnosing PPLs since the 1970s [1-3]. However, the accuracy of diagnosing PPLs using FB under X-ray fluoroscopic guidance is reportedly $14-71 \%[1-8]$. One factor that potentially limits the diagnostic accuracy of the standard bronchoscope is lesion size, as lesions $<2 \mathrm{~cm}$ have very low yields ranging $11-42 \%$ [6-10]. Some respiratory physicians prefer to diagnose small PPLs from tissue samples obtained by percutaneous needle aspiration cytology or biopsy [11-15]. Although success rates of these techniques might be very high, with 76-97\% diagnostic accuracy [6, 15], these techniques have several problems. First, they have the potential to spread malignant cells from the tumour into the pleural cavity $[11,12]$. For patients with poor pulmonary function, these techniques result in an increased risk of pneumothorax. Moreover, systemic arterial air embolism is a rare but severe complication $[13,14]$.

Therefore, in many institutions, patients with small peripheral pulmonary lesions undergo surgical biopsy procedures, such as video-assisted thoracic surgery, although such invasive procedures are undesirable for elderly patients and patients with poor respiratory function. In order to spare patients from these operative procedures, new imaging and guidance technology is necessary.

Recently developed small-calibre ultrasound probes have enabled wider clinical application of ultrasonography (US) to tracheal and bronchial lesions. Guided by FB, a small-calibre
US probe can be successfully introduced into the trachea and bronchus for assessment and diagnosis of endobronchial lesions or tumour metastasis to the mediastinal or hilar lymph nodes [16-18]. Transbronchial needle aspiration under endobronchial ultrasonography (EBUS) guidance has improved the $\mathrm{N}$-staging yields for lung cancer without clear endoscopic landmarks [17-20]. EBUS has also been applied to the assessment of PPLs [16, 21]. KuRIMOTO et al. [22] analysed EBUS images with the aim of distinguishing between benign and malignant disease, identifying the type of lung cancer, and determining the degree of differentiation. Moreover, HerTh et al. [23] applied EBUS as imaging guidance for transbronchial biopsy (TBB) of PPLs. In the present study, the EBUS was further combined with the guide-sheath (GS) technique. Biopsy forceps covered with a GS can be moved to the lesions under EBUS guidance, after which biopsy and brushing specimens can be sequentially obtained by keeping the GS in the lesion. The present study was undertaken in order to examine the usefulness of a new technique, EBUS with GS (EBUS-GS), as a guide for TBB and bronchial brushing cytology for diagnosis of PPLs.

\section{Methods}

\section{Patients}

Between December 1, 2002, and July 31, 2003, at Hokkaido University Medical Hospital (Sapparo, Japan), and Iwamizawa 

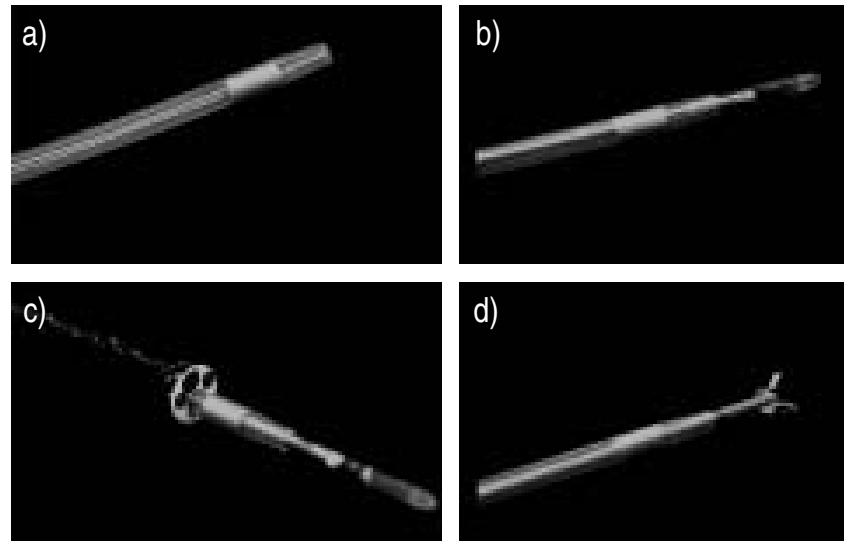

Fig. 1.- Ultrasound probe and guide-sheath for endobronchial ultrasonography (US) with guide-sheath (GS). The GS a) is attached to the US probe b). The US probe with GS is inserted through the working channel of the fibreoptic bronchoscope c). Biopsy forceps are also inserted into a GS d).

Municipal General Hospital (Iwamizawa, Japan), 24 patients with 24 PPLs $\leqslant 30 \mathrm{~mm}$ in mean diameter referred for diagnostic bronchoscopy were enrolled. PPLs were defined as those that were surrounded by pulmonary parenchyma and not visible by bronchoscopy (no evidence of endobronchial lesion, extrinsic compression, submucosal tumour, or narrowing, inflammation or bleeding of the bronchus). All chest computed tomographs (CT) were reviewed and the mean diameters of the PPLs were recorded. After written informed consent, patients underwent the bronchoscopic procedure.

\section{EBUS-GS-guided TBB and bronchial brushing}

Each patient was pre-medicated using $15 \mathrm{mg}$ of pentazocine hydrochloride and $0.5 \mathrm{mg}$ of atropine sulphates as a routine procedure for TBB in the two hospitals described above. Local anaesthesia of the upper respiratory tract was achieved using 4\% lidocaine. EBUS was performed using an endoscopic ultrasound system (EU-M30S; Olympus, Tokyo,
Japan), equipped with a $20-\mathrm{MHz}$ mechanical radial-type probe (XUM-S20-17R; Olympus), having an external diameter of $1.4 \mathrm{~mm}$ (fig. 1). FBs with a working channel of $2.0 \mathrm{~mm}$ in diameter were used (BF-P-260F, BF-P-240, BF-P200; Olympus). The probe was inserted into a GS, and the GS-covered probe was inserted through the working channel of the FB and advanced to the peripheral pulmonary lesion in order to obtain an EBUS image. The probe and GS were confirmed to have reached the lesion by EBUS imaging and X-ray fluoroscopy. After localising the lesion using EBUS imaging, the probe was removed and the GS remained in the peripheral lesion. Biopsy forceps and a bronchial brush were introduced via the GS to perform pathological and cytological examination (fig. $2 \mathrm{a}-\mathrm{c}$ ). When the lesion was not identified on the EBUS image, a double-hinged curette was inserted into the GS, and the appropriate bronchus was selected by manipulating the curette. The curette could be flexed and rotated by the proximal handle of the curette. When the lesion was not identified on the EBUS image or next to the EBUS image, using standard procedure, the GS was pulled, the next bronchus in the proximal position was selected by curetteloaded GS and the curette was manipulated. Once the bronchus was determined, the curette was removed. The probe was then inserted through the GS and the acquired EBUS images were again examined. These procedures were repeated by trial-and-error method until the probe and GS were found to have reached the correct position (fig. $2 \mathrm{~d}-\mathrm{g}$ ).

\section{Results}

\section{Patient data}

A total of 24 patients (10 males, 14 females) with an average age of $67.5 \pm 14.8$ yrs (range $24-83$ yrs) were examined. The mean diameter of the PPLs was $18.4 \pm 6.3 \mathrm{~mm}$ (8.0-27.5 mm). Localisation of the PPLs was the right upper lobe in five patients $(20.8 \%)$, the right middle lobe in three patients $(12.5 \%)$, the right lower lobe in 10 patients $(41.7 \%)$ and the left upper lobe in six patients $(25.0 \%)$. There were no PPLs examined in the left lower lobe. a)

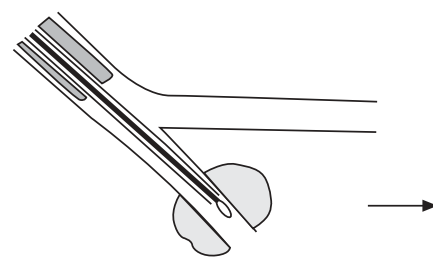

d)

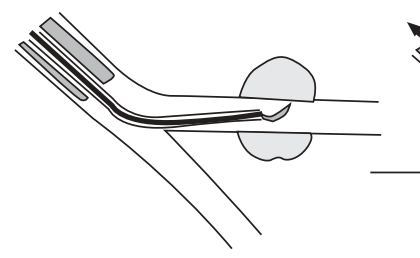

b)

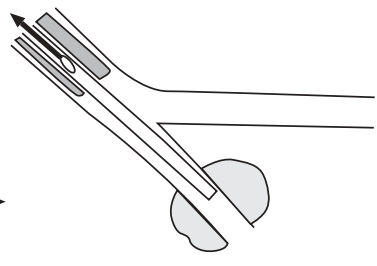

e)

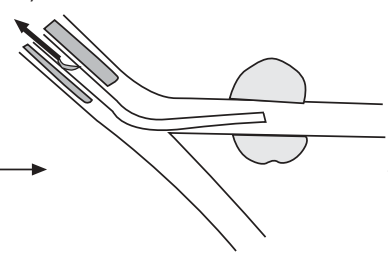

f)

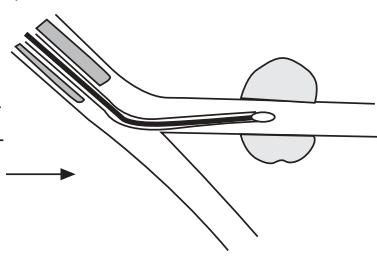

C)

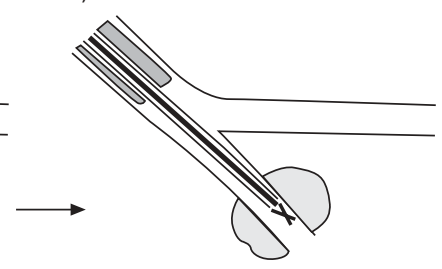

g)

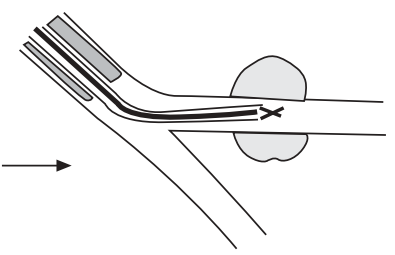

Fig. 2.-Endobronchial ultrasonography (EBUS)-guide-sheath (GS)-guided transbronchial biopsy (TBB). a) EBUS probe with GS is advanced to the peripheral pulmonary lesion via fibreoptic bronchoscope. After confirmation by EBUS imaging, b) the US probe is pulled out, and c) TBB and bronchial brushing are performed via the GS. When the lesion is not identified by EBUS imaging, d) a curette is inserted into the GS and the appropriate bronchus is selected. e) The curette is then pulled out and f) the EBUS probe is again inserted into the GS to perform EBUS imaging. After confirmation by EBUS imaging, g) TBB and bronchial brushing are performed. 


\section{EBUS-GS-guided transbronchial biopsy and bronchial brushing}

In 19 patients $(79.2 \%)$ EBUS detected the PPL. In 10 patients the EBUS probe reached the peripheral lesions with curette navigation. All patients whose PPLs were visible by EBUS imaging subsequently underwent EBUS-GS-guided TBB and bronchial brushing. The mean number of specimens

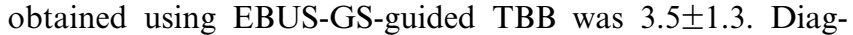
nosis was made in 14 cases $(58.3 \%) ; 10$ cases of primary lung cancer, one case of metastatic lung cancer of the thyroid, one case of metastatic lung cancer of the uterus and two cases of benign disease (pneumonia, one case; pneumoconiosis, one case). Pathological diagnosis was performed in 11 lesions $(45.8 \%)$ and cytological diagnosis was performed in 11 lesions $(45.8 \%)$ (table 1). A total of five lesions that were visible by EBUS and for which diagnoses could not be obtained had insufficient biopsy and brush samples taken. When evaluating the position of EBUS against the 19 lesions visible by EBUS, the US probe was located in the bronchus inside the lesions in 14 cases (12 malignant diseases, two benign diseases). In 13 of these cases, cytopathological diagnosis was obtained. The US probe was located in the bronchus adjacent to the lesion in five cases (two malignant diseases, three benign diseases), and in one of these malignant cases cytopathological diagnosis was obtained.

A representative case is shown in figure 3. An 82-yr-old male who underwent right upper lung lobectomy for pulmonary adenocarcinoma and who had thyroid carcinoma 12 yrs previously was admitted to one of the participating hospitals with an abnormal chest shadow. Chest radiograph and CT showed a pulmonary nodule of $8 \mathrm{~mm}$ in diameter in the left S3a. EBUS showed a low-echoic nodule surrounded by a strong reflected interface produced between the aerated lung and the lesion. Metastatic adenocarcinoma of the thyroid was diagnosed by EBUS-GS-guided TBB.

In 10 patients with 10 lesions, the EBUS-GS-guided bronchoscopic procedure was nondiagnostic. Seven of these 10 lesions were diagnosed as malignant diseases by repeated conventional bronchoscopic procedures using thicker forceps or a thicker bronchial brush, by CT-guided TBB using an ultrathin bronchoscope and navigation by virtual bronchoscopy or by surgical resection. In the other three lesions, the size of the lesions was reduced and these lesions were designated as benign.

When evaluated according to size (table 1), 15 lesions were $<20 \mathrm{~mm}$ in mean diameter. Ten lesions $(66.7 \%)$ were visible on EBUS images and eight lesions $(53.3 \%)$ were diagnosed using this procedure (six lesions by pathological diagnosis and five lesions by cytological diagnosis). In nine lesions with mean diameters of $20-30 \mathrm{~mm}$, all lesions were visible on EBUS images and six lesions $(66.7 \%)$ were diagnosed (five lesions by pathological diagnosis and six lesions by
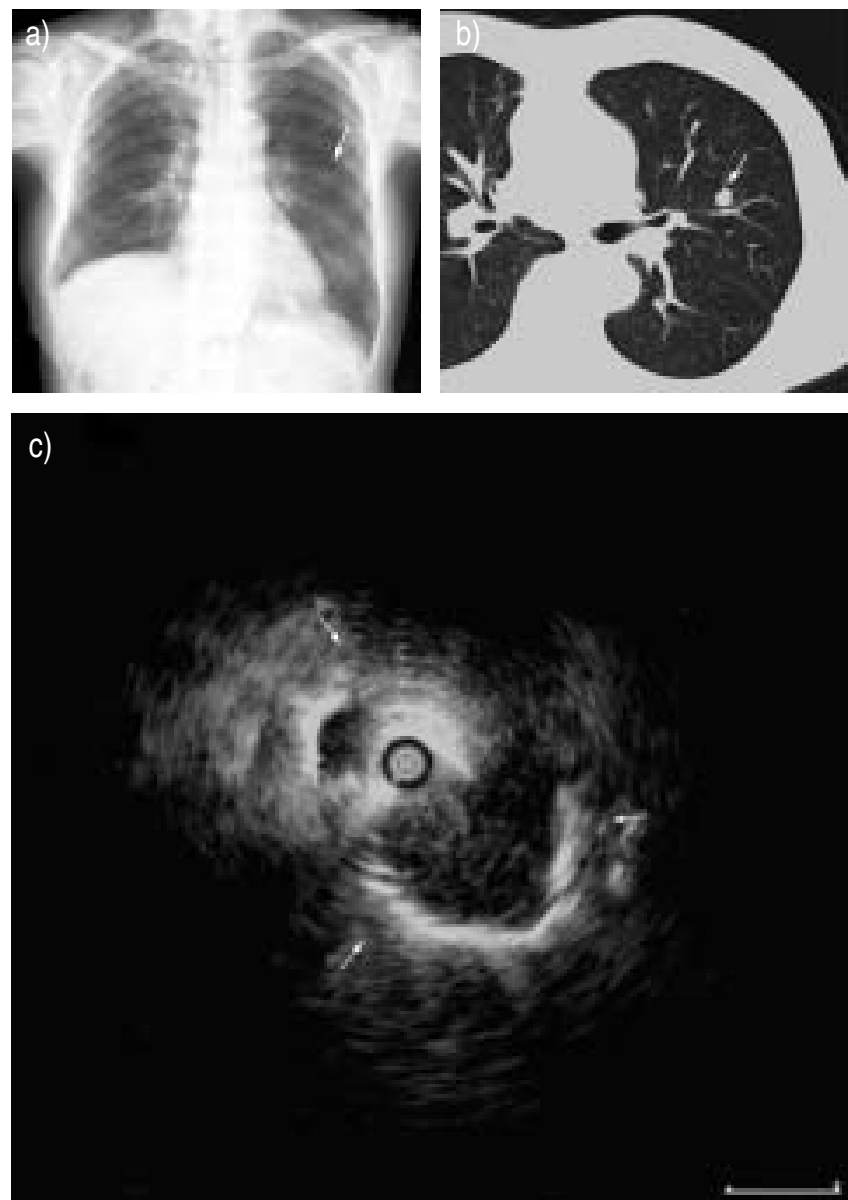

Fig. 3.-An 82-yr-old male who underwent right upper lung lobectomy for pulmonary adenocarcinoma and who had thyroid carcinoma 12 yrs previously was admitted to the study hospital with an abnormal chest shadow. a) Chest radiograph and b) computed tomography showed a pulmonary nodule of $8 \mathrm{~mm}$ in diameter in the left S3a (arrows). c) Endobronchial ultrasonography showed a lowechoic nodule surrounded by a strong reflected interface produced between the aerated lung and the lesion (arrowheads; scale bar $=0.5 \mathrm{~cm}$ ). Metastatic adenocarcinoma of the thyroid was diagnosed by EBUS-guide-sheath-guided transbronchial biopsy.

cytological diagnosis). The diagnostic accuracy of the EBUSGS-guided bronchoscopic procedure in malignant and benign lesions was $66.7 \%$ and $33.3 \%$, respectively.

When restricted to malignant lesions, the diagnostic sensitivity for a lesion $<20 \mathrm{~mm}$ in mean diameter was $60 \%$. There were no significant differences in diagnostic sensitivity of EBUS-GS with regard to bronchial segment.

Table 1.-Diagnostic yield of endobronchial ultrasonography (EBUS)-guide sheath (GS)

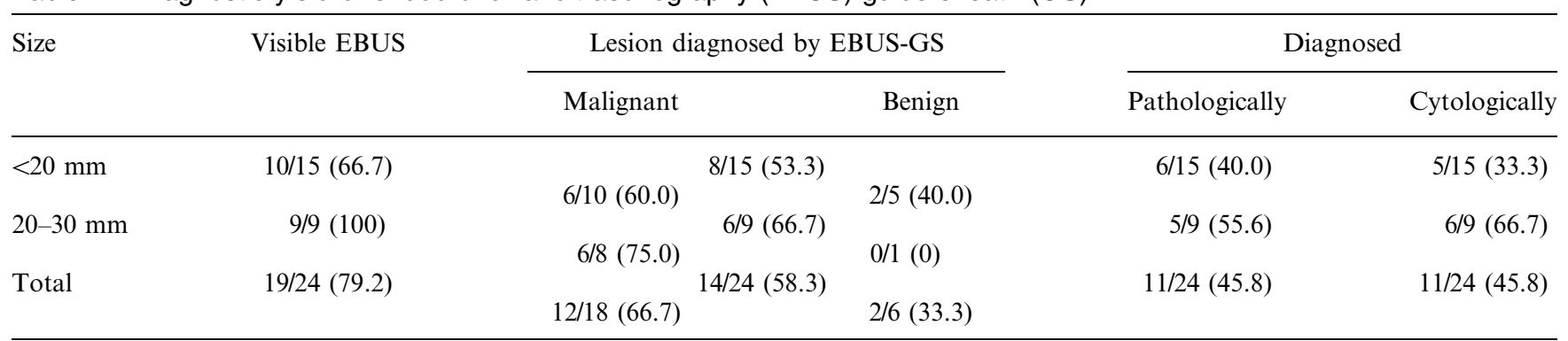

Data are presented as number of lesions/total lesions (\%). 


\section{Complications}

One patient developed pneumothorax $(4.2 \%)$ which was treated by tube thoracostomy. No major bleeding occurred during the diagnostic procedure.

\section{Discussion}

The endobronchial application of US was first described in 1990 [24]. To date, several reports have shown the usefulness of EBUS for identifying PPLs [16, 17, 21-23]. The air content of lung parenchyma completely reflects the US signal. Pulmonary masses have a hypoechoic texture when compared with the surrounding tissue, and have sharply defined borders due to the strong reflective interface produced between the aerated lung and the lesions [25]. HURTER and HANRATH [16] reported the successful visualisation of peripheral lung lesions in 19 out of 26 cases and GolDBERG et al. [17] reported that EBUS provided unique information in 18 out of 25 cases (including six peripheral lesions and 19 hilar tumours). KURIMOTO et al. [22] have classified the EBUS patterns of the internal structure of PPLs and speculated on the histology of the lesions based on EBUS pattern.

The present study has demonstrated that EBUS-GS-guided TBB and bronchial brushing were useful for evaluating PPLs. EBUS imaging shows the precise location of the pulmonary lesion and EBUS-GS enables diagnostic specimens to be obtained from the most appropriate position. The combination of EBUS and the curette-loaded GS technique has enabled visualisation of PPLs that are not accessible with a probe and allowed biopsy specimens to be obtained from PPLs that are not accessible by regular techniques. In the current study, 10 lesions were accessible with the use of the curette. Moreover, three lesions could not be detected by Xray fluoroscopy in the present report, of which one lesion was diagnosed as adenocarcinoma by EBUS-GS. The diagnostic sensitivity of EBUS-GS-guided TBB and bronchial brushing for diagnosing PPLs $\leqslant 30 \mathrm{~mm}$ in mean diameter was $58.3 \%$ in the present study. When restricted to lesions having a mean diameter of $<20 \mathrm{~mm}$, the diagnostic sensitivity of this procedure was as high as $53.3 \%$, and $40.0 \%$ of the lesions were diagnosed pathologically. The accuracy of diagnosing PPLs $<20 \mathrm{~mm}$ in diameter using FB under X-ray fluoroscopy has been reported to be a very low, ranging $11-42 \%$ [6-10]. At the participating institute in this study, the diagnostic sensitivity of FB for small PPLs $<20 \mathrm{~mm}$ in diameter under $\mathrm{X}$-ray fluoroscopic guidance over the past year was $35 \%$ and the rate for obtaining diagnostic biopsy samples was as low as $13 \%$ (data not shown). Two cases of benign disease (one pneumoconiosis and one pneumonia) and two case of metastatic lung cancer were diagnosed. In these four cases, unnecessary surgical procedures could have been circumvented as a result of the diagnosis obtained by EBUS-GS.

Several improvements may be necessary in order to increase the yield of EBUS-GS-guided TBB and bronchial brushing. In the present study, $79.2 \%$ of PPLs were detected by EBUS imaging. However, five lesions $(20.8 \%)$ that were visible by EBUS could not be diagnosed using the EBUS-GS-guided bronchoscopic procedure. This is largely because the biopsy forceps and bronchial brushes are small (diameter $1.2 \mathrm{~mm}$ ) and sufficient specimens could not be obtained. In addition, the position of EBUS may affect the result. In the present study, out of 14 cases in which the US probe was located in the bronchus inside the lesion, 13 cases were diagnosed cytopathologically. In contrast, out of five cases in which the US probe was located in the bronchus adjacent to the lesion, only one case was diagnosed cytopathologically.
The authors of the present study normally experienced $<1 \mathrm{~mL}$ of bleeding and coagulated blood was occasionally trapped in the GS, but bleeding outside of the GS was hardly seen even when the GS was removed. It is assumed that the wedged GS in the bronchus leading to the biopsied lesion is useful for stopping bleeding.

GS dislocation is occasionally encountered. The GS was occasionally dislocated from the target bronchus when the US probe or biopsy forceps was pulled out strongly from the GS or when a patient drew a deep breath. GS dislocation could be minimised when the procedure was performed with the bronchoscope wedging into the subsegment bronchus. Even in these undiagnosed lesions, the EBUS images were extremely useful to identify the appropriate bronchi during subsequent conventional bronchoscopic procedures using thicker forceps and bronchial brushes.

The benefits of EBUS-GS for TBB and bronchial brushing are as follows: 1) to confirm the precise location of PPLs by EBUS imaging even when such lesions are not visible on X-ray fluoroscopy; 2) to facilitate obtaining biopsy and brushing specimens repeatedly by leaving the GS in the PPLs; 3) to obtain biopsy specimens from PPLs that are accessible only through the use of a curette via the GS; 4) to decrease bleeding resulting from trapping the GS in the bronchus; and 5) to assess the internal structure of PPLs. Points 2), 3) and 4) are additional values of the GS technique above EBUS alone.

In summary, endobronchial ultrasonography with guidesheath-guided transbronchial biopsy and bronchial brushing was performed safely and was found to be effective for diagnosing peripheral pulmonary lesions. The endobronchial ultrasonography and guide-sheath technique is a very promising new modality for diagnosing small peripheral pulmonary lesions. A randomised, prospective study of diagnosing peripheral pulmonary lesions and examining cost effectiveness by bronchoscopy, with or without endobronchial ultrasonography with guide-sheath, is currently being planned.

\section{References}

1. Zavala DC. Diagnostic fiberoptic bronchoscopy: techniques and results of biopsy in 600 patients. Chest 1975; 68: 12-19.

2. Kvale PA, Bode FR, Kini S. Diagnostic accuracy in lung cancer: comparison of techniques used in association with flexible fiberoptic bronchoscopy. Chest 1976; 69: 752-757.

3. Cortese DA, McDougall JC. Biopsy and brushing of peripheral lung cancer with fluoroscopic guidance. Chest 1979; 75: 141-145.

4. Chechani V. Bronchoscopic diagnosis of solitary pulmonary nodules and lung masses in the absence of endobronchial abnormality. Chest 1996; 109: 620-625.

5. Mori K, Yanase N, Kaneko M, Ono R, Ikeda S. Diagnosis of peripheral lung cancer in cases of tumors $2 \mathrm{~cm}$ or less in size. Chest 1989; 95: 304-308.

6. Schreiber G, McCrory DC. Performance characteristics of different modalities of suspected lung cancer: summery of published evidence. Chest 2003; 123: Suppl. 1, 115s-128s.

7. Baaklini WA, Reinoso MA, Gorin AB, Sharafkaneh A, Manian P. Diagnostic yield of fiberoptic bronchoscopy in evaluating solitary pulmonary nodules. Chest 2000; 117: 1049-1054.

8. Stringfield JT, Markowitz DJ, Bentz RR, Welch MH, Weg JG. The effect of tumor size and location on diagnosis by fiberoptic bronchoscopy. Chest 1977; 72: 474 476.

9. Gasparini S, Ferretti M, Secchi EB, Baldelli S, Zuccatosta L, Gusella P. Integration of transbronchial and pericutaneous approach in the diagnosis of peripheral pulmonary nodules 
or masses: experience with 1,027 consecutive cases. Chest 1995; 108: 131-137.

10. Naidich DP, Sussman R, Kutcher WL, Aranda CP, Garay SM, Ettenger NA. Solitary pulmonary nodules: CT-bronchoscopic correlation. Chest 1988; 93: 595-598.

11. Sawabata N, Ohta M, Maeda H. Fine-needle aspiration cytologic technique for lung cancer has a high potential of malignant cell spread through the tract. Chest 2000; 118: 936-939.

12. Seyfer AE, Walsh DS, Graeber GM, Nuno IN, Eliasson AH. Chest wall implantation of lung cancer after thin-needle aspiration biopsy. Ann Thorac Surg 1989; 48: 284-286.

13. Mokhlesi B, Ansaarie I, Bader M, Tareen M, Boatman J. Coronary artery air embolism complicating a CT-guided transthoracic needle biopsy of the lung. Chest 2002; 121: 993-996.

14. Wong RS, Ketai L, Temes RT, Follis FM, Ashby R. Air embolus complicating transthoracic percutaneous needle biopsy. Ann Thorac Surg 1995; 59: 1010-1011.

15. Tsukada H, Satou T, Iwashima A, Souma T. Diagnostic accuracy of CT-guided automated needle biopsy of lung nodules. Am J Roentgenol 2000; 175: 239-243

16. Hurter T, Hanrath P. Endobronchial sonography: feasibility and preliminary results. Thorax 1992; 47: 565-567.

17. Goldberg BB, Steiner RM, Liu JB, et al. US-assisted bronchoscopy with use of miniature transducer-containing catheters. Radiology 1994; 190: 233-237.
18. LeBlanc JK, Espada R, Ergun G. Non-small cell lung cancer staging techniques and endoscopic ultrasound. Chest 2003; 123: $1718-1725$.

19. Shannon JJ, Bude RO, Orens JB, et al. Endobronchial ultrasound-guided needle aspiration of mediastinal adenopathy. Am J Respir Crit Care Med 1996; 153: 1424 1430 .

20. Kurimoto N, Murayama M, Yoshioka S, Nishisaka T, Inai K, Dohi K. Assessment of usefulness of endobronchial ultrasonography in determination of depth of tracheobronchial tumor invasion. Chest 1999; 115: 1500-1506.

21. Omori S, Yakiguchi $\mathrm{Y}$, Hiroshima $\mathrm{K}$, et al. Peripheral pulmonary diseases: Evaluation with endobronchial USInitial experience. Radiology 2002; 224: 603-608.

22. Kurimoto N, Murayama M, Yoshioka S, Nishisaka T. Analysis of the internal structure of peripheral pulmonary lesions using endobronchial ultrasonography. Chest 2002; 122: $1887-1894$.

23. Herth FJF, Ernst A, Becker HD. Endobronchial ultrasoundguided transbronchial lung biopsy in solitary pulmonary nodules and peripheral lesions. Eur Repir J 2002; 20: 972974.

24. Hurter T, Hanrath P. Endobronchiale sonographie zur diagnostik pulmonaler und mediastinaler tumoren. Dtsch Med Wochenschr 1990; 115: 1899-1905.

25. Falcone F, Fois F, Grosso D. Endobronchial Ultrasound. Respiration 2003; 70: 179-194. 